\title{
UNIMODULAR COMMUTATORS
}

\author{
MORRIS NEWMAN
}

(Communicated by Thomas H. Brylawski)

\begin{abstract}
Let $R$ be a principal ideal ring and $M_{k, n}$ the set of $k \times n$ matrices over $R$. The following statments are proved: (a) If $k \leq n / 3$ then any primitive element of $M_{k, n}$ occurs as the first $k$ rows of the commutator of two elements of $\mathrm{SL}(n, R)$. (b) If every element of $\operatorname{SL}(3, R)$ is the product of at most $c_{3}$ commutators, then every element of $\operatorname{SL}(n, R)$ is the product of at most $c_{n}$ commutators, where $c_{n}<c \log n+c_{3}-3, c=2 \log (3 / 2)=4.932 \ldots$, and $n \geq 3$. (c) If $n \geq 3$, then every element of $\operatorname{SL}(n, Z)$ is the product of at most $c \log n+40$ commutators, where $c$ is given in (b) above
\end{abstract}

1. Introduction. Let $R$ denote an arbitrary principal ideal ring, and let $M_{k, n}=M_{k, n}(R)$ denote the set of $k \times n$ matrices over $R, M_{n}=M_{n}(R)$ the set of $n \times n$ matrices over $R$. If $1 \leq k \leq n$, an element $\alpha$ of $M_{k, n}$ is said to be primitive if $d_{k}(\alpha)=1$, where $d_{k}(\alpha)$ is the $k$ th determinantal divisor of $\alpha$. As usual, let $\Gamma=\mathrm{SL}(n, R)$ denote the multiplicative group of matrices of $M_{n}$ of determinant 1. The primitive elements of $M_{k, n}$ for $1 \leq k<n$ are just those which may be completed to an element of $\Gamma$.

It is well known that in many cases $\Gamma$ coincides with its commutator subgroup $\Gamma^{\prime}$. This happens, for example, when $R$ is a euclidean ring of characteristic not 2, and $n \geq 3$. Assume from now on that $\Gamma=\Gamma^{\prime}$. The question considered here is whether or not every element of $\Gamma$ may be represented as the product of a bounded number of commutators, where the bound depends only on $n$. Accordingly, when $\Gamma$ has this property, we define $c_{n}=c_{n}(R)$ as the least positive integer such that every element of $\Gamma$ is the product of at most $c_{n}$ commutators. In order to complete the definition, we define $c_{n}$ to be infinity, when $\Gamma$ does not have this property.

In this paper we reduce the question to the case $n=3$, and prove that the answer is in the affirmative for $R=Z$ and $n \geq 3$. The result in this case depends on a crucial theorem of D. Carter and G. Keller (see [1]) who prove that if $A$ is any matrix of the form

$$
\left[\begin{array}{lll}
1 & 0 & 0 \\
0 & a & b \\
0 & c & d
\end{array}\right]
$$

belonging to $\operatorname{SL}(3, Z)$, then $A$ is the product of at most 41 elementary matrices. (An elementary matrix of $\Gamma$ is any matrix of the form $I+x E_{i j}$, where $I=I_{n}$ is the $n \times n$ identity matrix, $i, j$ are distinct integers such that $1 \leq i, j \leq n, E_{i j}$ is the $n \times n$ matrix with a 1 in position $(i, j)$ and 0 elsewhere, and $x$ is any element of $R$.) Since every elementary matrix is also a commutator, the result cited above

Received by the editors April 15, 1986 and, in revised form, September 26, 1986. 1980 Mathematics Subject Classification (1985 Revision). Primary 15A36, $20 \mathrm{H} 05$. 
implies that any matrix of the form

$$
\left[\begin{array}{lll}
1 & 0 & 0 \\
0 & a & b \\
0 & c & d
\end{array}\right]
$$

belonging to $\mathrm{SL}(3, Z)$ is the product of at most 41 commutators.

The bound we obtain is surprisingly small -in fact of order $\log n$ - and prompts the conjecture that every element of $\operatorname{SL}(n, Z)$ is the product of at most $c$ commutators for some absolute constant $c$, provided that $n \geq 3$. The case $n=2$ is an exception, since then $\left(\Gamma: \Gamma^{\prime}\right)=12$.

2. Statement of results. We prove three theorems. The first is a completion theorem which generalizes Theorem 6 of [2], and the others are concerned with bounds for $c_{n}$.

THEOREM 1. Let $\alpha$ be a primitive element of $M_{k, n}$, where $k \leq n / 3$. Then matrices $A, B$ of $\mathrm{SL}(n, R)$ exist such that $\alpha$ coincides with the first $k$ rows of $[A, B]=A B A^{-1} B^{-1}$.

THEOREM 2. Suppose that $n \geq 3$. Then

$$
c_{n}(R)<c \log n+c_{3}(R)-3
$$

where

$$
c=2 / \log (3 / 2)=4.932 \ldots
$$

THEOREM 3. Suppose that $n \geq 3$. Then every element of $\mathrm{SL}(n, Z)$ is the product of at most $c \log n+40$ commutators, where $c$ is given by (2).

2. Proofs.

Proof of THEOREM 1. Write $\alpha=[U V]$, where $U \in M_{k}$ and $V \in M_{k, n-k}$. Let $S$ be any element of $\mathrm{SL}(n-k, R)$. We have

$$
\left[\begin{array}{cc}
I_{k} & 0 \\
0 & S^{-1}
\end{array}\right] \quad\left[\begin{array}{cc}
U & V \\
*
\end{array}\right] \quad\left[\begin{array}{cc}
I_{k} & 0 \\
0 & S
\end{array}\right]=\left[\begin{array}{cc}
U & V S \\
& *
\end{array}\right] .
$$

Thus it is sufficient to prove the theorem for $[U V S]$, since conjugates of commutators are commutators. Choose $S$ so that $V S=[W 0]$, where $W \in M_{k}$ and 0 is a $k \times(n-2 k)$ block of zeros. Since $k \leq n / 3$, it is sufficient to prove the theorem for [ $U W 0$ ], where now 0 is a $k \times k$ block of zeros.

Since $[U W]$ is a primitive element of $M_{k, 2 k}$, matrices $X, Y$ exist which belong to $M_{k}$ such that

$$
\left[\begin{array}{ll}
U & W \\
X & Y
\end{array}\right] \in \mathrm{SL}(2 k, R)
$$

Put

$$
\left[\begin{array}{ll}
U & W \\
X & Y
\end{array}\right]^{-1}=\left[\begin{array}{ll}
U_{1} & W_{1} \\
X_{1} & Y_{1}
\end{array}\right]
$$


We note that if $I$ stands for the $k \times k$ identity matrix, then

We now have

$$
\begin{gathered}
A=\left[\begin{array}{lll}
0 & 0 & I \\
I & 0 & 0 \\
0 & I & 0
\end{array}\right] \in \mathrm{SL}(3, R), \\
B=\left[\begin{array}{ccc}
U_{1} & W_{1} & 0 \\
X_{1} & Y_{1} & 0 \\
0 & 0 & I
\end{array}\right] \in \mathrm{SL}(3 k, R) .
\end{gathered}
$$

$$
\begin{aligned}
{[A, B] } & =A B A^{-1} B^{-1} \\
& =\left[\begin{array}{lll}
0 & 0 & I \\
I & 0 & 0 \\
0 & I & 0
\end{array}\right]\left[\begin{array}{ccc}
U_{1} & W_{1} & 0 \\
X_{1} & Y_{1} & 0 \\
0 & 0 & I
\end{array}\right]\left[\begin{array}{lll}
0 & I & 0 \\
0 & 0 & I \\
I & 0 & 0
\end{array}\right]\left[\begin{array}{ccc}
U & W & 0 \\
X & Y & 0 \\
0 & 0 & I
\end{array}\right] \\
& =\left[\begin{array}{ccc}
0 & 0 & I \\
U_{1} & W_{1} & 0 \\
X_{1} & Y_{1} & 0
\end{array}\right]\left[\begin{array}{ccc}
X & Y & 0 \\
0 & 0 & I \\
U & W & 0
\end{array}\right]=\left[\begin{array}{ccc}
U & W & 0 \\
* & * & * \\
* & * & *
\end{array}\right] .
\end{aligned}
$$

This completes the proof.

For the remaining theorems, two lemmas are required.

LEMMA 1. Suppose that $n \geq 2$. Then a matrix $C$ exists such that both $C$ and $C-I$ belong to $\mathrm{SL}(n, R)$.

PROOF. Let $q(x)$ be any monic polynomial of degree $n-2$ belonging to $R[x]$, and let $p(x)=x(x-1) q(x)+(-1)^{n}$. Let $C$ be the companion matrix of $p(x)$. Since $\operatorname{det}(C)=(-1)^{n} p(0)=1, C$ belongs to $\operatorname{SL}(n, R)$. The characteristic polynomial of $C-I$ is $p(x+1)$; and since $\operatorname{det}(C-I)=(-1)^{n} p(1)=1, C-I$ also belongs to $\mathrm{SL}(n, R)$. This completes the proof.

LEMMA 2. Suppose that $n \geq 3$, and let

$$
E=\left[\begin{array}{cc}
I_{r} & 0 \\
A & I_{s}
\end{array}\right] \in \mathrm{SL}(n, R),
$$

where $A$ is any element of $M_{s, r}$. Then $E$ is a commutator.

PrOOF. Either $r$ or $s$ must be $\geq 2$; we may assume that $s \geq 2$. Let $C \in \operatorname{SL}(s, R)$ be such that $C-I$ also belongs to $\operatorname{SL}(s, R)$ (Lemma 1). Let $B$ be an element of $M_{s, r}$ to be determined later. We have

$$
\begin{aligned}
& {\left[\begin{array}{ll}
I & 0 \\
B & 1
\end{array}\right]\left[\begin{array}{ll}
I & 0 \\
0 & C
\end{array}\right]\left[\begin{array}{cc}
I & 0 \\
-B & I
\end{array}\right]\left[\begin{array}{cc}
I & 0 \\
0 & C^{-1}
\end{array}\right]} \\
& \quad=\left[\begin{array}{ll}
I & 0 \\
B & C
\end{array}\right]\left[\begin{array}{cc}
I & 0 \\
-B & C^{-1}
\end{array}\right]=\left[\begin{array}{cc}
I & 0 \\
(I-C) B & I
\end{array}\right] .
\end{aligned}
$$

Since $I-C$ is unimodular, we may choose $B=(I-C)^{-1} A$. For this $B, E$ becomes a commutator, and the proof is concluded.

PROOF OF THEOREM 2. Let $L$ be any matrix of $\operatorname{SL}(n, R)$. Put $k=[n / 3]$. By Theorem 1 , there is a matrix $K$ of $\operatorname{SL}(n, R)$ such that the first $k$ rows of $K$ coincide with the first $k$ rows of $L^{-1}$, and $K$ is a commutator. Then

$$
K L=\left[\begin{array}{cc}
I_{k} & 0 \\
A & L_{1}
\end{array}\right],
$$


where $A \in M_{n-k, k}$ and $L_{1} \in \mathrm{SL}(n-k, R)$. Thus

$$
L=K^{-1}\left[\begin{array}{cc}
I_{k} & 0 \\
A & L_{1}
\end{array}\right]=K^{-1}\left[\begin{array}{cc}
I_{k} & 0 \\
A & I_{n-k}
\end{array}\right]\left[\begin{array}{cc}
I_{k} & 0 \\
0 & L_{1}
\end{array}\right]
$$

By Lemma 2, the matrix

$$
\left[\begin{array}{cc}
I_{k} & 0 \\
A & I_{n-k}
\end{array}\right]
$$

is a commutator, and so $L$ has been expressed as the product of two commutators and the matrix $\left[\begin{array}{ll}I_{K} & 0 \\ 0 & L_{1}\end{array}\right]$ where the order of $L_{1}$ is $n-[n / 3]$.

We now repeat this process, obtaining the sequence of integers

$$
\begin{aligned}
& n_{0}=n \\
& n_{1}=n_{0}-\left[n_{0} / 3\right] \\
& \quad \ldots \\
& n_{k+1}=n_{k}-\left[n_{k} / 3\right]
\end{aligned}
$$

Since $n_{k}=3\left[n_{k} / 3\right]+r_{k}$, where $r_{k}=0,1,2$, we have the inequalities

$$
\frac{2}{3} n_{k} \leq n_{k+1} \leq \frac{2}{3} n_{k}+\frac{2}{3}, \quad k=0,1, \ldots,
$$

which readily imply that

$$
\left(\frac{2}{3}\right)^{k} n \leq n_{k}<\left(\frac{2}{3}\right)^{k} n+2, \quad k=0,1, \ldots
$$

We now choose $k$ so that $3 \leq\left(\frac{2}{3}\right)^{k} n,\left(\frac{2}{3}\right)^{k} n+2 \leq 7$, which is equivalent to

$$
\begin{gathered}
\frac{n}{5} \leq\left(\frac{3}{2}\right)^{k} \leq \frac{n}{3} \\
\frac{\log (n / 5)}{\log (3 / 2)} \leq k \leq \frac{\log (n / 3)}{\log (3 / 2)} .
\end{gathered}
$$

Since

$$
\frac{\log (n / 3)}{\log (3 / 2)}-\frac{\log (n / 5)}{\log (3 / 2)}=\frac{\log (5 / 3)}{\log (3 / 2)}>1,
$$

such a $k$ exists. It then follows that $L$ is the product of $2 k-2$ commutators, and a matrix of the form $\left[\begin{array}{cc}I_{n-r} & 0 \\ 0 & T\end{array}\right]$, where $T \in \mathrm{SL}(r, Z)$ and $r=3,4,5,6$. Employing this reduction again for $r=4,5,6$ we find that $T$ is the product of at most $c_{3}+s$ commutators, where $s=0$ for $r=3, s=2$ for $r=4, s=4$ for $r=5$, and $s=4$ for $r=6$. It follows that in all cases, an upper bound for $c_{n}$ is given by $2 k-2+c_{3}+4=2 k+c_{3}+2$. Since $k \leq \log (n / 3) / \log (3 / 2)$, we have that

$$
\begin{aligned}
c_{n} & \leq \frac{2 \log n}{\log (3 / 2)}+c_{3}+2-\frac{2 \log 3}{\log (3 / 2)} \\
& <\frac{2 \log n}{\log (3 / 2)}+c_{3}-3,
\end{aligned}
$$

the desired inequality. This completes the proof.

PROOF OF THEOREM 3. Theorem 3 follows directly from Theorem 2 and the result of $\mathrm{D}$. Carter and $\mathrm{G}$. Keller cited above, since $c_{3}(Z) \leq 43$. 
4. Some open questions. A number of interesting open problems are suggested by these results:

(a) Suppose that $G$ is a finitely presented perfect group. When is it possible to deduce from the presentation of $G$ alone that an absolute constant $c$ exists such that every element of $G$ is the product of at most $c$ commutators? This property of a group is of importance for classification purposes, and the results of this paper should be of use whenever $G$ has a faithful representation as a subgroup of $\operatorname{SL}(n, Z)$ for some $n$.

(b) The commutator subgroup $\operatorname{SL}^{\prime}(2, Z)$ of $\operatorname{SL}(2, Z)$ is of index 12 , and is a free group of rank 2. Somewhat more generally, let $G=\{x, y\}$ be a free group of rank 2 , freely generated by $x$ and $y$. Then if $k$ is a positive integer, the element $z=\left(x y x^{-1} y^{-1}\right)^{k}$ is the product of $k$ commutators. Prove or disprove that $z$ is not the product of fewer than $k$ commutators.

(c) Determine the perfect subgroups of finite index of $\mathrm{SL}(n, Z)$.

The author thanks the referee for correcting some typographical errors, and for suggesting the inclusion of the section above.

\section{BIBLIOGRAPHY}

1 D. Carter and G. Keller, Elementary expressions for unimodular matrices, Comm. Algebra 12 (1984), 379-389.

2 M. Newman, Matrix completion theorems, Proc. Amer. Math. Soc. 94 (1985), 39-45.

Department of Mathematics, University of California, Santa Barbara, CALIFORNIA 93106 